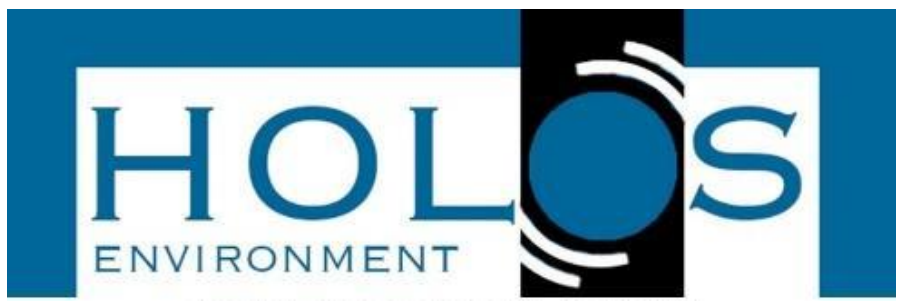

\title{
ESTIMATIVAS DE CONDUTIVIDADE HIDRÁULICA A PARTIR DE PERFILAGENS GEOFÍSICAS NO SISTEMA AQUÍFERO GUARANI
}

\section{HYDRAULIC CONDUCTIVITY DERIVED FROM GEOPHYSICAL LOGGING IN THE GUARANI AQUIFER SYSTEM}

\author{
Bruno Zanon Engelbrecht ${ }^{1}$; Elias Hideo Teramoto'; Roger Dias Gonçalves ${ }^{1}$; \\ Hung Kiang Chang ${ }^{2}$
}

Artigo recebido em: 16/01/2020 e aceito para publicação em: 06/02/2020. DOI: http://dx.doi.org/10.14295/holos.v20i1.12369

Resumo: A condutividade hidráulica $(K)$ é um parâmetro essencial para fins distintos no estudo de águas subterrâneas, tais como para avaliação da produtividade e vulnerabilidade de aquíferos, gerenciamento de recursos hídricos subterrâneos, estimativas da velocidade de migração de plumas de contaminação e simulações numéricas de fluxo e transporte. Embora existam diferentes técnicas para obtenção dos valores de condutividade hidráulica, apenas um número reduzido possibilita determinar, com elevada resolução, suas variações em porções profundas em sub-superfície. Na tentativa de solucionar esta deficiência, o presente trabalho desenvolveu e testou uma nova abordagem, na qual os valores de porosidade, estimados a partir de perfis sônico e de raios gama, foram utilizados para calcular os valores de $\mathrm{K}$ com elevada resolução espacial, empregando o modelo de Kozeny-Carman. Esses testes foram conduzidos em um poço profundo (1242 m) que explora água do Sistema Aquífero Guarani. Os valores médios de K obtidos são próximos dos obtidos por testes de bombeamento. Verificou-se também que a Formação Pirambóia apresenta fortes variações de K, enquanto a Formação Botucatu é relativamente homogênea, o que está em concordância com o contexto deposicional dessas duas unidades. Por fim, foi possível confimar que os valores de $\mathrm{K}$ obtidos a partir dos testes de bombeamento representam a média geométrica dos valores de $\mathrm{K}$ ao longo da seção filtrante dos poços testados.

Palavras-chave: Condutividade hidráulica. Perfilagem geofísica. Sistema Aquífero Guarani. Teste de bombeamento. Kozeny-Carman.

\begin{abstract}
Hydraulic conductivity $(\mathrm{K})$ is a crucial parameter for different applications in groundwater studies, such as vulnerability and aquifer assessment, groundwater management, contamination velocity estimation, and numerical simulations of groundwater flow and transport. Although there are diverse approaches in order to obtain $\mathrm{K}$ values, only a small number of these methods allow determination of high-resolution $\mathrm{K}$ changes in great aquifer depths. In an attempt to solve this difficulty, the present work developed and tested a new approach, in which porosity values, estimated from sonic and gamma ray logs, were used to calculate $\mathrm{K}$ values with high spatial availability by using the Kozeny-Carman model. These tests were conducted in a deep well (1242 m), which exploits the Guarani Aquifer System water. The mean K values found by the

${ }^{1}$ LEBAC - Laboratório de Estudo de Bacias (UNESP), Campus de Rio Claro, SP. CEA - Centro de Estudos Ambientais Unesp - Campus de Rio Claro. E-mails: (bruno.engelbrecht@unesp.br, elias.hideo-teramoto@unesp.br, roger.dias@unesp.br)

2 DGA - Departamento de Geologia Aplicada (UNESP), Campus de Rio Claro, SP. LEBAC - Laboratório de Estudo de Bacias, Unesp - Campus de Rio Claro, SP. E-mail: (chang.hung-kiang@unesp.br)
\end{abstract}


proposed approach were similar to $\mathrm{K}$ value derived from pumping test. From the use of this approach, it was noted that Pirambóia formation presents strong $\mathrm{K}$ variability, while Botucatu formation is relatively homogeneous, in accordance with the depositional context of these two geological units. Finally, we confirm that $\mathrm{K}$ values obtained from the pumping tests represent the geometric mean of the $\mathrm{K}$ values along the filtered section of tested wells.

Keywords: Hydraulic conductivity. Geophysical logging. Guarani Aquifer System. Pumping tests. KozenyCarman.

\section{INTRODUÇÃO}

O parâmetro condutividade hidráulica (K) é uma propriedade física que descreve a facilidade com que um fluido pode se mover através de interstícios porosos em solos e rochas (FETTER, 2018). É dependente da permeabilidade intrínseca do material, do grau de saturação e da densidade e viscosidade do fluido. A condutyividade hidráulica é um parâmetro fundamental para o entendimento das potencialidades e limites de explotação de aquíferos, assim como para previsões baseadas em modelos matemáticos, estimativas da velocidade de migração de plumas de contaminação e ações para correta gestão dos recursos hídricos sub-superficiais.

Existem diversas técnicas para determinação de $\mathrm{K}$ e, em geral, se baseiam em ensaios laboratoriais, testes de campo ou modelos empíricos. Entretanto, os valores obtidos oferecem resultados distintos devido às variações de $\mathrm{K}$, em função das heterogeneidades geológicas, e à escala de análise, crescendo linearmente com 0 incremento desta e tornando-se constante a partir de um determinado ponto (ROVEY e CHERKAUER, 1995; SCHULZE-MAKUCH et al., 1999; DEWANDEL et al., 2012).

$\mathrm{Na}$ atualidade, a Equação de Kozeny-Carman representa o modelo empírico mais difundido para determinação da permeabilidade e, consequentemente, da condutividade hidráulica de aquíferos. A consistência das estimativas de $\mathrm{K}$ a partir da porosidade, empregando a Equação de Kozeny-Carman ou uma versão modificada desta, foi demonstrada em diversos trabalhos, incluindo McGregor (1965), Panda e Lake (1994), Koponen et al. (1997), Pape et al. (2000) e Carrier III (2003). Embora a Equação de Kozeny-Carman seja amplamente difundida, ela requer dados de porosidade obtidas a partir de ensaios com amostras indeformadas, o que dificulta sua obtenção em intervalos profundos. Entretanto, a porosidade é um parâmetro que pode estimado a partir de 
perfilagem geofísica, como demonstrado por Wyllie (1956), Raymer et al. (1980), Han et al. (1986), Freund (1992) e Vernik (1994), dentre outros.

Os testes de bombeamento representam a metodologia mais usual e difundida para determinação da condutividade hidráulica de aquíferos (SUN, 2018). Estes consistem na extração de volumes constantes de água do poço, enquanto o rebaixamento do nível d'água é monitorado. A análise da curva de rebaixamento em função do tempo permite a estimativa de propriedades hidráulicas do aquífero, dentre as quais a transmissividade, que representa o produto da condutividade hidráulica pela espessura saturada do aquífero (FETTER, 2018). Segundo Raghavan et al. (2004), nas últimas décadas ocorreu um grande progresso nos modelos e na metodologia dos testes de bombeamento e, embora sejam apropriados para estudos regionais, os valores de $\mathrm{K}$ assim obtidos não permitem avaliar as variações de condutividade hidráulica ao longo do perfil filtrante do poço, de forma que não é possível aferir o efeito das heterogeneidades no regime de fluxo. McCall e Christ (2010) apresentaram a técnica Hydraulic Profiling Tolls (HPT) como uma alternativa para obtenção de valores de K com elevada resolução espacial. Entretanto, a aplicabilidade dessa técnica é restrita às porções rasas do aquífero.

Com o objetivo de solucionar as deficiências assinaladas, o presente trabalho apresenta, em caráter preliminar, uma metodologia para obtenção de $\mathrm{K}$ a partir do modelo semi-empírico de Kozeny-Carman, empregando dados de porosidade estimados a partir de perfis sônico e de raios gama. A adequabilidade do modelo proposto foi testada a partir da comparação com testes de bombeamento. Adicionalmente, buscou-se avaliar se as estimativas de permeabilidade fornecidas pela equação de Kozeny-Carman e pelo teste de bombeamento oferecem resultados comparáveis entre si, a despeito da escala de análise.

\section{MATERIAIS E MÉTODOS}

\section{1 Área de estudo}

O poço P2 localiza-se no município de Colômbia (SP), pertencente a mesorregião 
de Ribeirão Preto (SP). O município é banhado pelas águas do Rio Grande e faz divisa com Barretos (SP), a sul, Guaíra (SP), a leste, e os municípios de Planura (MG) e Frutal (MG), a norte (Figura 1). O poço $P 2$ foi perfurado em área rural e a captação de água do Sistema Aquífero Guarani (SAG) é destinada para irrigação de pomares de laranja.

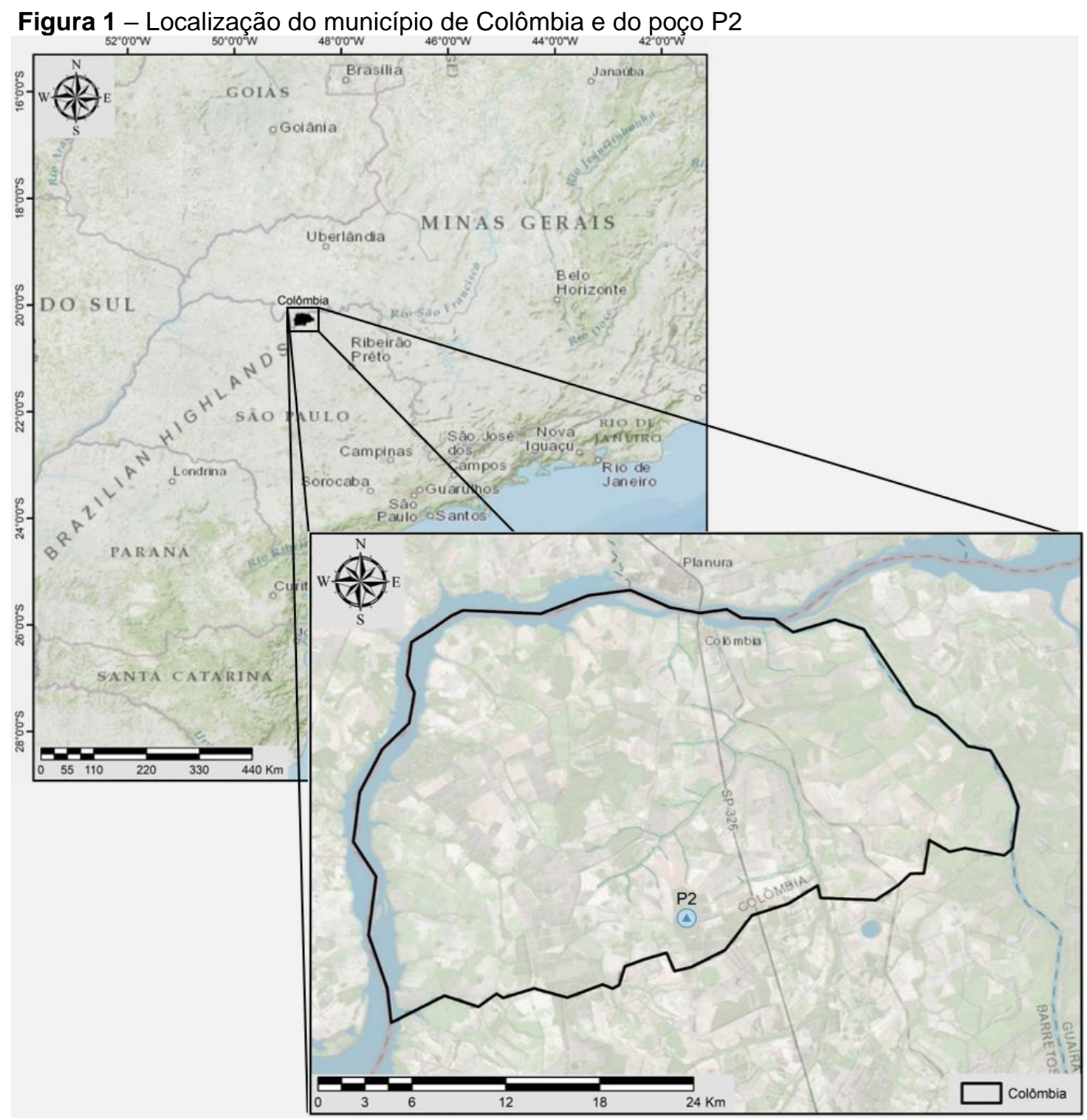

Fonte: Elaborado pelos autores

\subsection{Perfil construtivo e geológico do poço}

A perfuração do poço P2 foi realizada pelo método rotativo, com circulação direta 
de fluido de perfuração composto essencialmente por bentonita. O perfil construtivo do poço tubular profundo é composto por selo sanitário, câmara de bombeamento e coluna de produção. A Figura 2 apresenta o perfil construtivo e geológico do poço P2.

Figura 2 - Perfil geológico e construtivo esquemático do poço P2

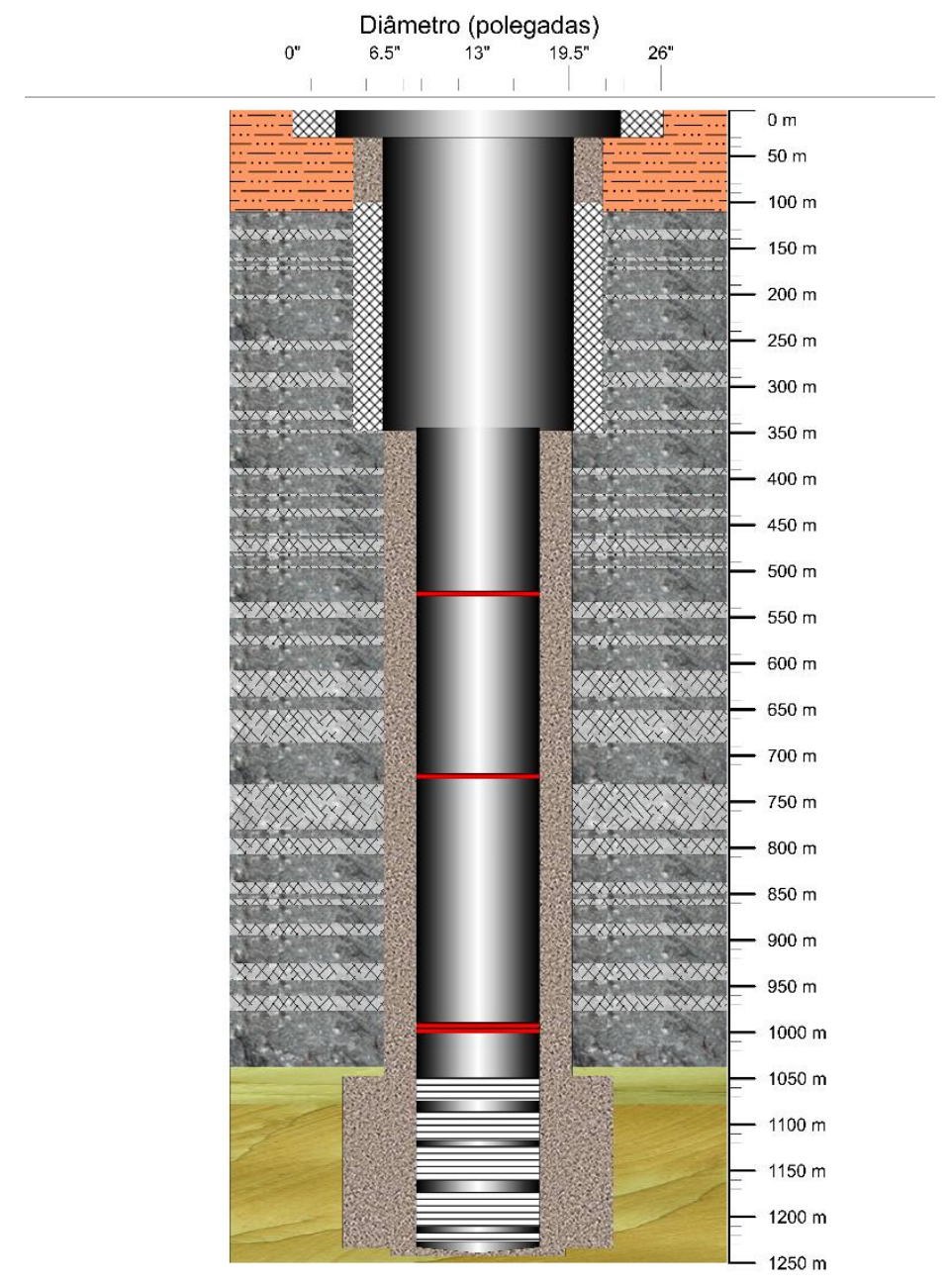

\begin{tabular}{|c|c|c|c|c|c|}
\hline \multicolumn{3}{|c|}{ Perfil Geológico } & \multicolumn{3}{|c|}{ Perfil Construtivo } \\
\hline$\cdots=$ & Gr. Bauru & Fm. Pirambóia & Tubo Liso & $\equiv$ & Filtros \\
\hline$x$ & Basalto Fraturado & Fm. Botucatu & Cimento & & Filtro Índice \\
\hline E & Basalto & & Pré-filtro & 11 & Ponteira \\
\hline
\end{tabular}

Fonte: Elaborado pelos autores

O perfil geológico do poço P2 foi estabelecido por meio da coleta, armazenamento e descrição das amostras de calha, coletadas em intervalos de dois metros; o poço atravessou rochas do Grupo Bauru, Formação Serra Geral, Formação Pirambóia e Formação Botucatu. 
No local, o Grupo Bauru apresenta espessura de 110,5 m e é composto por arenitos finos a muito finos, bem selecionados, vermelhos, atribuídos à Formação Adamantina. Na porção basal desse pacote foi identificado um siltito argiloso, provavelmente associado à Formação Araçatuba, com aproximadamente $40 \mathrm{~m}$ de espessura.

A partir de 110,5 m de profundidade ocorrem as rochas basálticas da Formação Serra Geral, que permanecem até $1036 \mathrm{~m}$ de profundidade, totalizando $925,5 \mathrm{~m}$ de espessura. Os basaltos ocorrem em seu estado de rocha sã e apresentam cor preta e cinza-escura. Foi registrada também a presença de zonas fraturadas, representadas por basaltos de coloração avermelhada, tempo de perfuração reduzido e presença de minerais argilosos preenchendo cavidades.

O Sistema Aquífero Guarani (SAG), composto pelas formações Pirambóia e Botucatu, ocorre a partir da profundidade de 1036 metros e permanece até a base do poço, em $1242 \mathrm{~m}$. Seu arcabouço é formado por arenitos finos a muito finos, moderadamente a bem selecionados, grãos predominantemente arredondados a subangulosos.

\subsection{Testes de bombeamento}

Os testes de bombeamento consistiram na extração ininterrupta de um volume constante de água por um período de 47 horas e 32 minutos, com medição da vazão e rebaixamento ao longo do tempo. O rebaixamento foi medido no próprio poço bombeado, utilizando-se um medidor elétrico graduado de nível d'água. A vazão do poço foi periodicamente mensurada com auxílio de um hidrômetro e cronômetro, de modo a avaliar as variações de vazão ao longo do teste. Após um período de aproximadamente 48 horas de teste, o bombeamento foi interrompido e a recuperação do nível no poço foi igualmente monitorada por 2 horas e 30 minutos.

Utilizando o aplicativo AQTESOLVE, foi realizado o ajuste das curvas do modelo às curvas de rebaixamento obtidas nos dois poços testados, empregando a solução de Theis (1935). Uma vez obtido o ajuste, os valores de transmissividade e armazenamento foram registrados. 


\subsection{Perfilagem geofísica}

As propriedades geofísicas das rochas no entorno do poço P2 foram registradas por meio de sensores de Raios Gama, Sônico, Potencial Elétrico Espontâneo e Resistividade, entre as profundidades de $350 \mathrm{~m}$ e $1242 \mathrm{~m}$. A interpretação dos perfis geofísicos restringiu ao intervalo onde ocorre o SAG, entre $1036 \mathrm{~m}$ e $1250 \mathrm{~m}$. Neste intervalo, os sensores empregados permitem identificar intervalos mais argilosos no trecho investigado.

\subsection{Estimativas de porosidade}

O perfil sônico permite estimar a porosidade do meio geológico. Uma vez que a velocidade de compressão do som no fluido é menor que a velocidade na rocha, a porosidade é uma grandeza inversamente propocional a velocidade sônica do meio (Wyllie et. al., 1956). Se houver espaço poroso na rocha e estiver cheio de fluido, a energia acústica levará mais tempo para passar do transmissor para o receptor. A velocidade registrada ou o tempo de viagem representa a soma da velocidade na rocha e no fluido que preenche seus interstícios.

Se os valores de velocidade de compressão do fluido e da rocha forem conhecidas, a porosidade pode ser estimada a partir do modelo empírico proposto por Wyllie (1956) e Raymer et al. (1980). Entretanto, como demonstrado em diversos trabalhos, tais como Han et al. (1986), Freund (1992) e Vernik (1994), a presença de argilas em arenitos promove o decréscimo na velocidade das ondas acústicas, induzindo a erros nas estimativas de porosidade. Han et al. (1986) propuseram um modelo empírico relacionando a velocidade da onda acústica compressional $\left(V_{p}\right)$ com a porosidade $(\emptyset)$ e o volume de argila $\left(\mathrm{V}_{\mathrm{Cl}}\right)$ (Equação 1):

$\emptyset=\frac{5,59-2,18 \cdot V_{c l}-V_{p}}{6,93}$ 
O teor de argila na rocha pode ser estimado a partir dos valores medidos de radiação gama $\left(G r_{l o g}\right)$ e valores de referência de radiação gama para arenitos $\left(G r_{\text {sand }}\right)$ e argilitos $\left(G r_{\text {shale }}\right)$ (Equação 2):

$V_{c l}=\frac{G r_{l o g}-G r_{\text {sand }}}{G r_{\text {shale }}-G r_{\text {sand }}}$

\subsection{Estimativas de permeabilidade}

A equação semi-empírica de Kozeny-Carman representa um dos modelos mais comumente empregados para estimativas de permeabilidade a partir de dados de porosidade. Este modelo foi desenvolvido por Kozeny (1927) e revisado por Carman (1938, 1956) (Equação 3):

$$
k=\frac{\emptyset^{3}}{C(1-\varnothing)^{2} S^{2}}
$$

Onde $\mathrm{k}, \emptyset, \mathrm{C}$ e $\mathrm{S}$ representam, respectivamente, a permeabilidade do meio, a porosidade, a constante de Kozeny e a área superficial específica baseada no volume dos sólidos.

O parâmetro $C$ pode ser calculado a partir da porosidade, empregando um modelo linear de tubos tridimensionais proposto por Mortensen et al. (1998) (Equação 4):

$$
C=\left\{4 \cos \left\{\frac{1}{3} \arccos (2 \varnothing-1)+\frac{4}{3} \pi\right\}+4\right\}^{-1}
$$

De acordo com Zhou e Houssain (2014), o parâmetro S, similarmente à constante C, pode ser estimado a partir da porosidade (Equação 5).

$\log (S)=a \cdot \log (\varnothing)+b$ 
Onde a e b são os coeficientes para os diferentes tipos de litologias; para arenitos limpos, a é -1,0 e b é -2,2, enquanto para arenitos argilosos, a é -2,5 e b é -2,3.

\section{RESULTADOS E DICUSSÃO}

\subsection{Teste de bombeamento}

Anteriormente ao início do teste de vazão máxima, o nível estático do poço foi aferido em 132,02 m de profundidade. Ao final de 47 horas e 32 minutos de bombeamento, o nível d'água estava em 203,15 m de profundidade, indicando um rebaixamento de $71,13 \mathrm{~m}$. O nível d'água mostrou tendência muito suave de rebaixamento, mantendo-se entre as profundidades de $202 \mathrm{~m}$ e $203 \mathrm{~m}$ por 29 horas contínuas de bombeamento (Figura 3). Ao longo do teste de bombeamento, a vazão do poço variou entre $387 \mathrm{~m}^{3} / \mathrm{h}$ e $419 \mathrm{~m}^{3} / \mathrm{h}$, sendo a vazão média de $404 \mathrm{~m} / \mathrm{h}$. As variações de vazão ao longo do teste representam até $4 \%$ da vazão média de bombeamento. Dividindo-se a vazão média de produção pelo rebaixamento do poço, calculou-se a vazão específica de $5,68 \mathrm{~m}^{3} / \mathrm{h} / \mathrm{m}$.

Figura 3 - Variação do nível d'água e vazão ao longo do teste de vazão máxima.

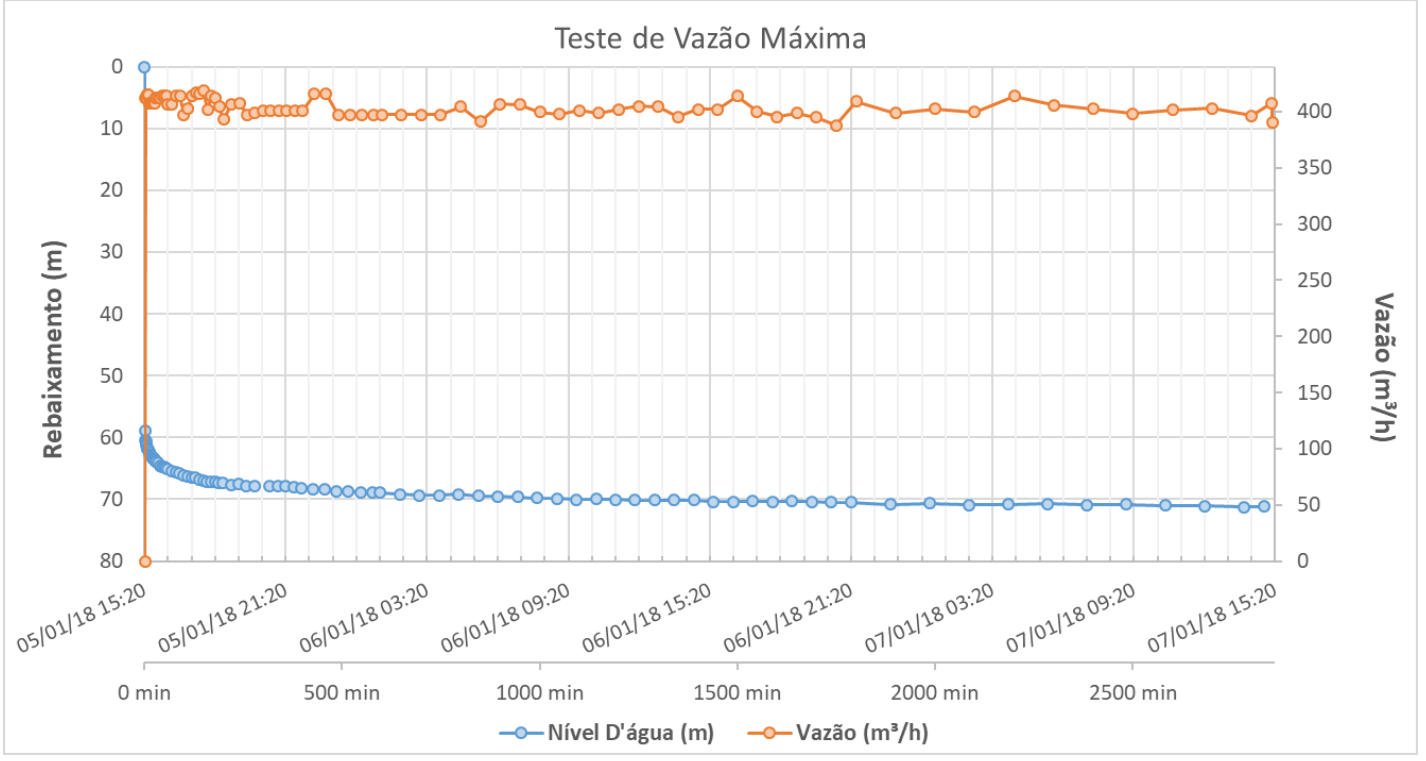

Fonte: Elaborado pelos autores 
O teste de recuperação foi iniciado imediatamente após a finalização do teste de vazão máxima. Após 2 horas e 30 minutos de recuperação, o nível d'água do poço foi aferido em 134,38 $\mathrm{m}$ de profundidade (rebaixamento de 2,36 $\mathrm{m}$ ), indicando a recuperação de $98,24 \%$ do nível d'água inicial (Figura 4).

Figura 4 - Resultados do teste de recuperação

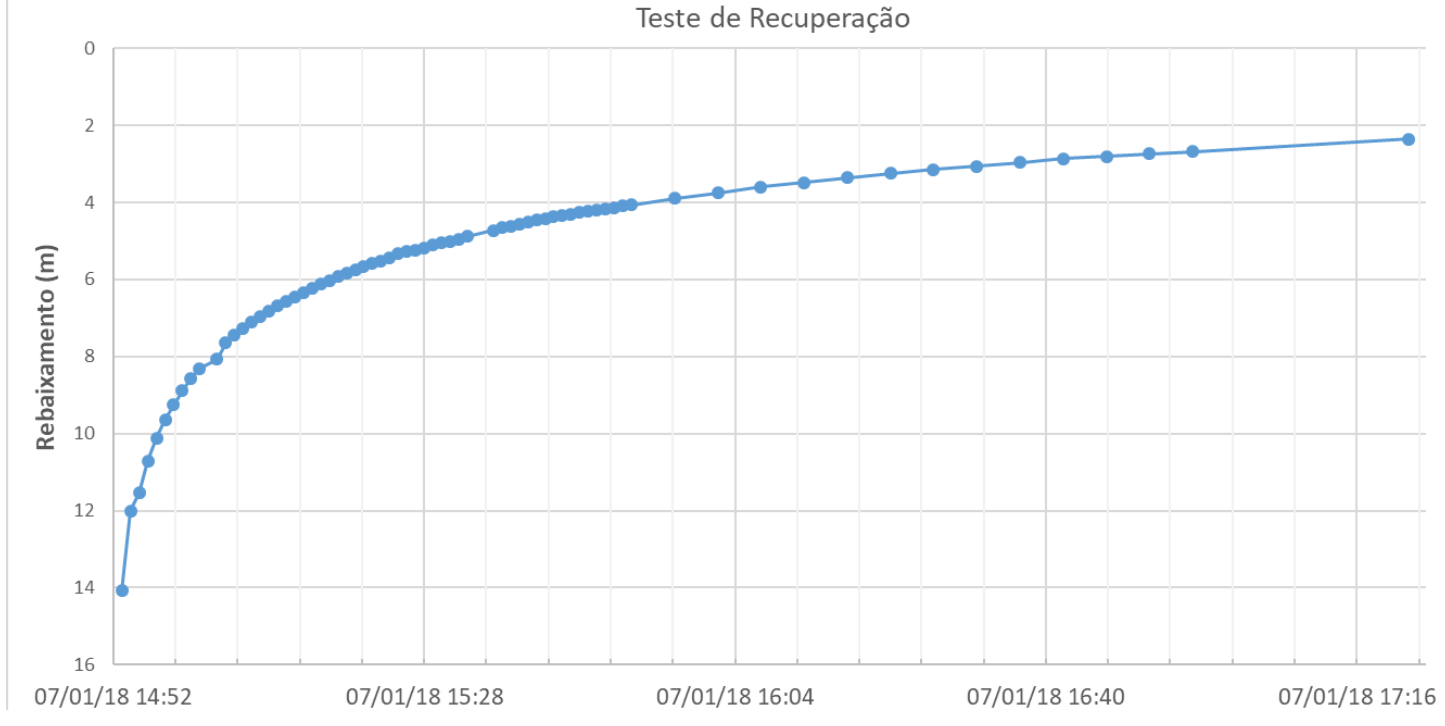

Fonte: Elaborado pelos autores

Com base nas curvas de rebaixamento e recuperação do poço, obtidas durante os testes de bombeamento preliminar e definitivo, foram estimados os parâmetros transmissividade (T) e K do aquífero com emprego do software AQTESOLVE, utilizandose o método de Theis (1935) para aquíferos confinados (Figura 5).

O valor de T do aquífero foi estimado entre $366,9 \mathrm{~m}^{2} / \mathrm{dia}$ e $374,8 \mathrm{~m}^{2} / \mathrm{dia}$, e o valor de $\mathrm{K}$ entre $2,23 \times 10^{-5} \mathrm{~m} / \mathrm{s}(1,93 \mathrm{~m} / \mathrm{dia})$ e $2,28 \times 10^{-5} \mathrm{~m} / \mathrm{s}(1,97 \mathrm{~m} / \mathrm{dia})$, considerando uma espessura saturada de $190 \mathrm{~m}$. O valor do coeficiente de armazenamento (S) não foi calculado, uma vez que estimativas seguras devem ser obtidas pelo monitoramento de poços de observação e não do próprio poço bombeado, como é o caso. 
Figura 5 - Estimativa de transmissividade utilizando o software AQTSOLVE e os dados de rebaixamento e recuperação do teste de bombeamento definitivo.

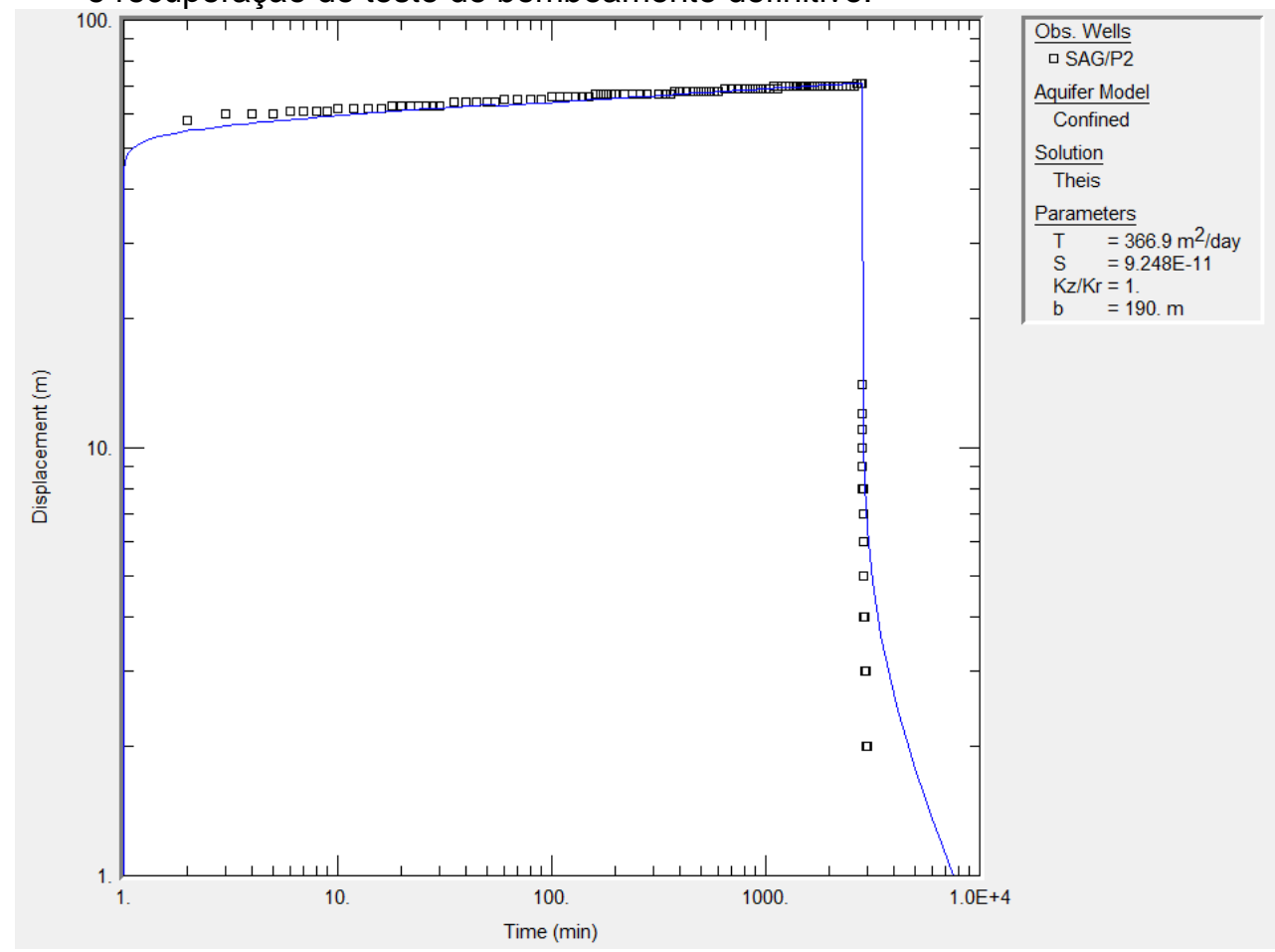

Fonte: Elaborado pelos autores

\subsection{Perfilagem Geofísica}

As propriedades geofísicas das rochas no entorno do poço P2, registradas pelos sensores de Raios Gama, Sônico, Potencial elétrico espontâneo e Resistividade, foram interpretadas no intervalo entre $1036 \mathrm{~m}$ e $1242 \mathrm{~m}$ de profundidade, correspondente as rochas do SAG (Figura 6).

O contato entre os basaltos e as rochas do SAG é delimitado pelo aumento abrupto do tempo de trânsito das ondas sonoras e do sinal dos raios gama, diminuição da resistividade das rochas, aumento no potencial espontâneo e diminuição no tempo de perfuração. O aumento do sinal de potencial espontâneo está relacionado ao aumento da permeabilidade dessas rochas, enquanto as variações de resistividade elétrica, tempo de trânsito das ondas acústicas e de raios gama estão às variações litológicas. 
Figura 6 - Perfis geofísicos no Sistema Aquífero Guarani. O contato entre a Formação Botucatu e a Formação Piramboia foi delimitado em 1080 m de profundidade. Perfis Raios Gama (GR), Sônico (DT), Potencial Elétrico Espontâneo (SP) e Resistividade (SN e DIR)

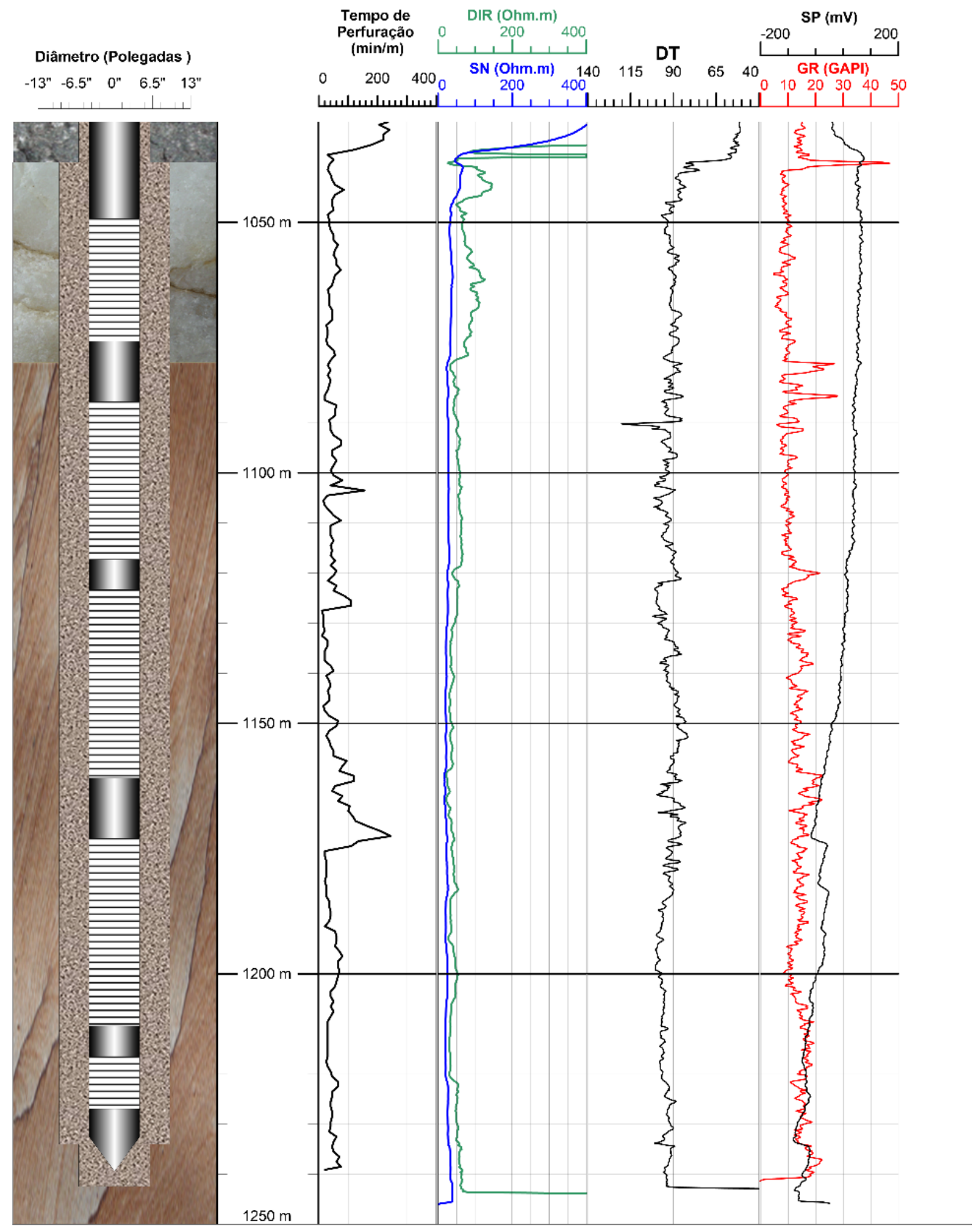

\section{Perfil Litológico}

$\begin{array}{ll}\ldots \text { Fr. Bauru } & \text { Fm. Pirambóia } \\ \text { Basalto Fraturado } & \text { Fm. Botucatu } \\ \text { Basalto }\end{array}$

Perfil Construtivo

Tubo Liso $\bar{\equiv}$ Filtros

Cimento Filtro Índice

Pré-filtro
(1) Ponteira

Fonte: Elaborado pelos autores 
No SAG, os perfis geofísicos permitiram diferenciar as formações Botucatu, no topo, e Piramboia, na base. A Formação Botucatu ocorre entre as profundidades de 1038 m e 1078 m e é identificada por valores de raios gama entre 7 e 10 na escala API e resistividades entre 32 ohm.m e 65 ohms.m. O contato entre as formações Botucatu e Piramboia é delimitado pela presença de material argiloso na profundidade de $1078 \mathrm{~m}$, quando o sinal dos raios gama atinge valores entre 20 e 25 na escala API, e as curvas de resistividade se aproximam entre si.

Na Formação Piramboia observa-se o aumento gradual do sinal de raios gama para a base da formação, diminuição da resistividade das rochas e do sinal de potencial espontâneo. A variação desses parâmetros petrofísicos reflete o aumento do conteúdo de argila nas rochas e a diminuição da permeabilidade do meio, típico desta unidade geológica. O sinal de raios gama permitiu identificar os trechos argilosos no SAG nas seguintes profundidades: $1077 \mathrm{~m}$ a $1080,5 \mathrm{~m} ; 1084 \mathrm{~m}$ a $1085 \mathrm{~m} ; 1118,5 \mathrm{~m}$ a $1122 \mathrm{~m}$; $1159 \mathrm{~m}$ a $1163 \mathrm{~m} ; 1164 \mathrm{~m}$ a $1166 \mathrm{~m} ; 1213 \mathrm{~m}$ a $1216 \mathrm{~m}$.

\subsection{Perfis de porosidade}

Os valores de $\Delta T$ obtidos a partir da perfilagem sônica foram convertidos em valores correspondentes de $\mathrm{Vp}$, que subsidiaram a elaboração do perfil porosidade do poço P2 (Figura 7), a partir da Equação 1. Os valores dos parâmetros $G r_{\text {sand }}$ e $G r_{\text {shale }}$ para o cálculo do parâmetro $V_{c l}$ foram, respectivamente, 4,79 API e 27,98 API, que representam os valores máximo e mínimo identificados na perfilagem de raios gama.

Cabe destacar que, no caso dos intervalos com argilitos/siltitos-argilosos, a porosidade estimada foi 0 , demonstrando que o modelo de Han et al. (1986) é inapropriado para litologias argilosas, prestando-se exclusivamente a litologias arenosas. A Tabela 1 apresenta uma análise estatística descritiva dos dados de porosidade estimados para as formações Pirambóia e Botucatu. Verifica-se que o valor médio de porosidade na Formação Botucatu (26\%) é superior àquele observado na Formação Pirambóia (22\%), sendo este fato diretamente associado a quantidades maiores de argilitos nos litotipos da Formação Pirambóia. Outro ponto a ser destacado é a ampla 
variação dos valores de porosidade na Formação Pirambóia, caracterizadas pelo elevado valor de desvio-padrão da porosidade (6\%) em oposição ao da Formação Botucatu (3\%).

Figura 7 - Perfil de porosidade determinado pelo modelo de Han et al. (1986), a partir dos dados do perfil sônico e de raios gama no poço P2

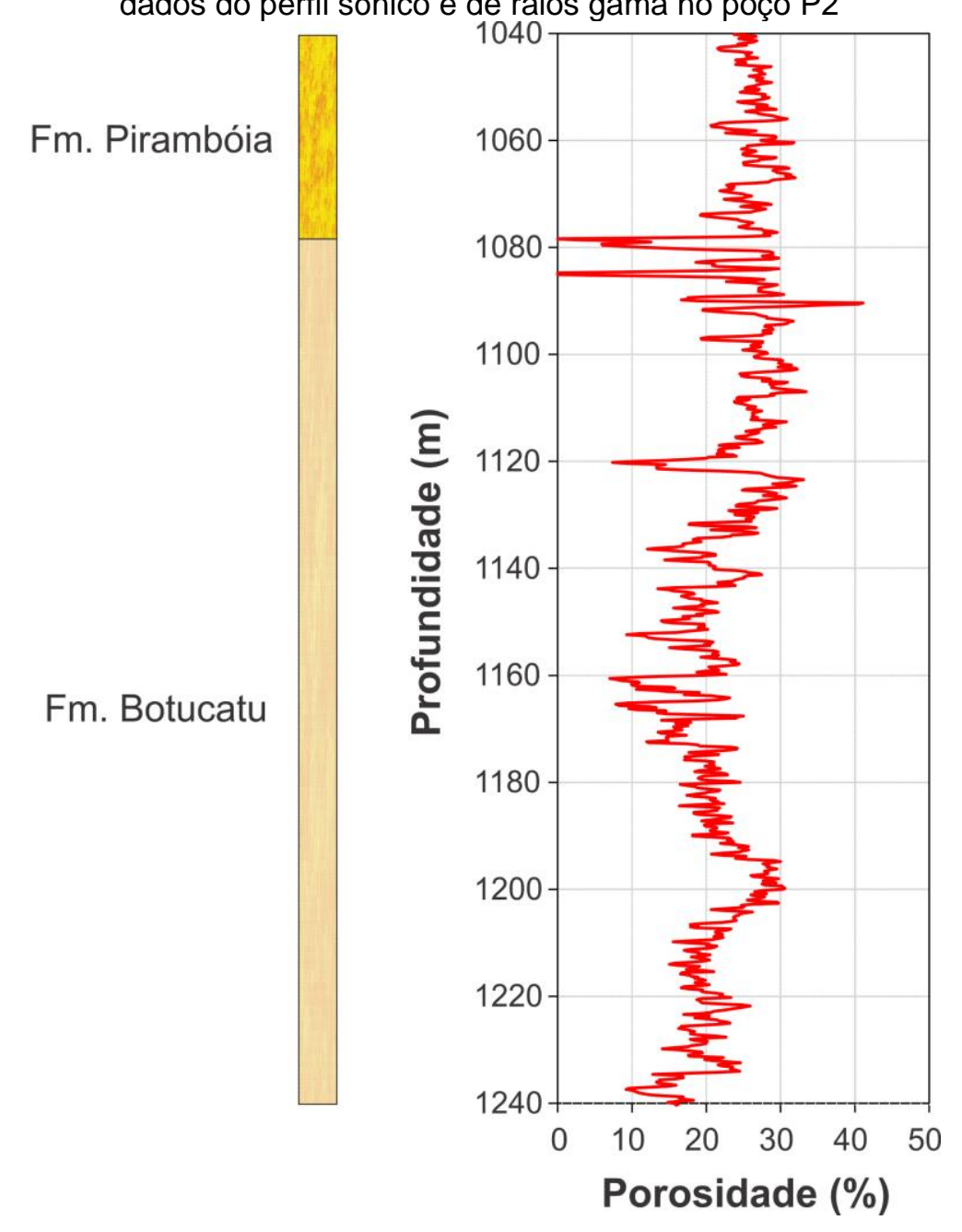

Fonte: Elaborado pelos autores

Tabela 1 - Análise estatística descritiva dos valores de porosidade estimados pelo modelo de Han et al. (1986), a partir da perfilagem sônica e de raios gama para as formações Pirambóia e Botucatu no poço P2

\begin{tabular}{ccc}
\hline Porosidade & Fm. Botucatu (\%) & Fm. Pirambóia (\%) \\
\hline Mínimo & 13 & 3 \\
Máximo & 32 & 41 \\
Média & 26 & 22 \\
Desvio padrão & 3 & 6 \\
\hline
\end{tabular}

Fonte: Elaborado pelos autores 
Os valores de porosidade estimados são similares àqueles de Araújo et al. (1999), que descrevem intervalos de 14 a 30\% e de Hirata et al. (2011), que reportam porosidades entre 20 e $40 \%$.

\subsection{Estimativa dos valores de condutividade hidráulica}

A partir do perfil de porosidade obtido a partir dos dados de perfilagem sônica e raios gama, empregando-se a Equação 1, foi construído o perfil de permeabilidade, empregando-se a Equação 2. Aplicando os dados de densidade e viscosidade da água a $57^{\circ} \mathrm{C}$, que corresponde à temperatura da água produzida no poço $\mathrm{P} 2$, foram obtidos os perfis com os valores correspondentes de condutividade hidráulica (Figura 8). São observadas fortes variações nos valores de condutividade hidráulica, particularmente no intervalo correspondente à Formação Pirambóia (1076,2 m a 1242 m), decorrentes da alternância de níveis com quantidades maiores ou menores de argila na matriz da rocha.

Figura 8 - Perfil de condutividade hidráulica determinada a partir do modelo de Kozeny-Carman, empregando-se os valores estimados de porosidade

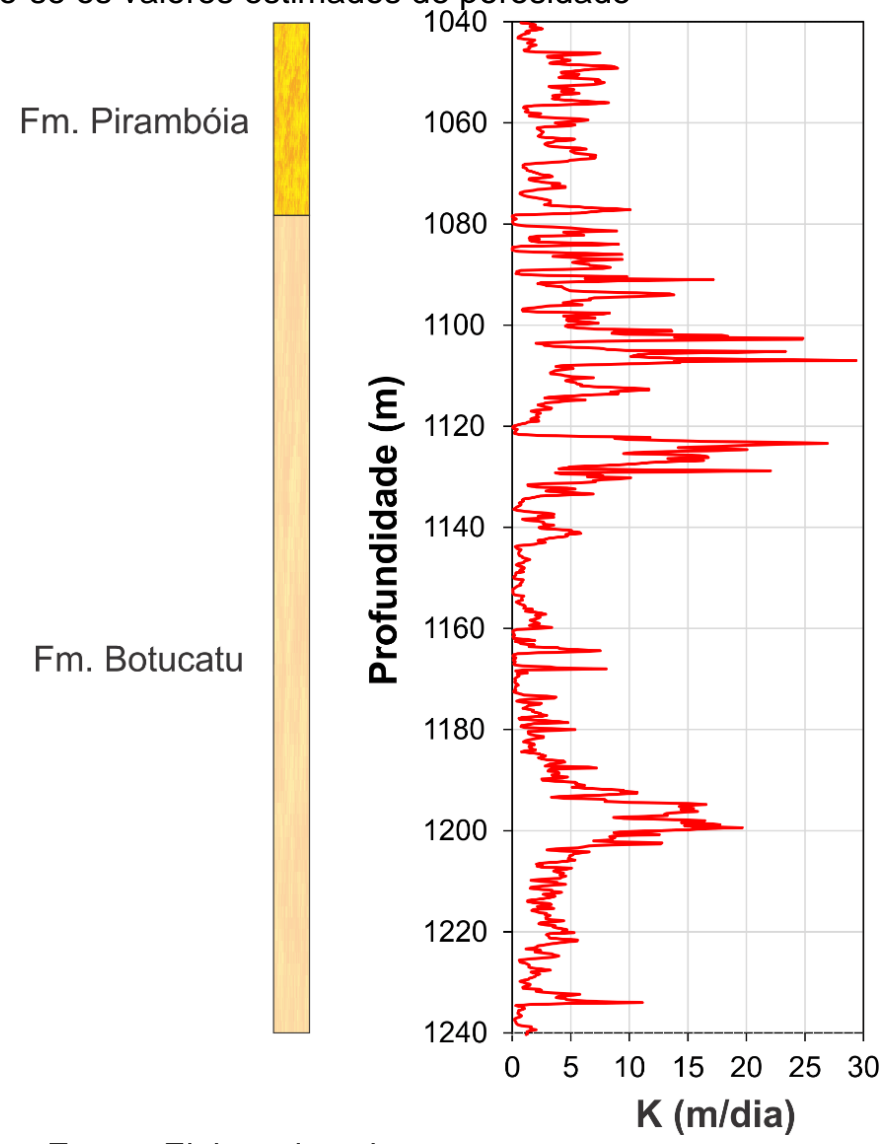

Fonte: Elaborado pelos autores 
A Tabela 2 apresenta a análise estatística dos dados de $\mathrm{K}$ estimados para as formações Pirambóia e Botucatu, demonstrando que as duas unidades possuem valores dissimilares. A Formação Botucatu apresenta um valor médio de $\mathrm{K}$ superior àquele estimado para a Formação Pirambóia, respectivamente, 3,60 m/dia e 2,79 m/dia. Observa-se ainda que os valores de $\mathrm{K}$ na Formação Pirambóia são bastante heterogêneos, variando dentro de um intervalo superior àquele observado na Formação Botucatu e, por esta razão, apresenta um desvio-padrão bastante superior. Destaca-se também que as elevadas variações de $\mathrm{K}$ identificadas na Formação Pirambóia são compatíveis com aquelas descritas por Höyng et al. (2014). Essas variações refletem a diversidade de condições de deposição flúvio-eólica na Formação Pirambóia (WU e CAETANO-CHANG, 1992; CAETANO-CHANG e WU, 2006), em oposição a um ambiente deposicional francamente eólico, atribuído à Formação Botucatu (WU e CAETANO-CHANG, 1992; GIANNINI et al., 2004).

Tabela 2 - Análise estatística descritiva dos valores de condutividade hidráulica estimados pelo modelo de Kozeny-Carman a partir dos dados de porosidade calculada através da perfilagem sônica e de raios gama para as Fm. Pirambóia e Botucatu no poço P2

\begin{tabular}{ccc}
\hline $\begin{array}{c}\text { Condutividade } \\
\text { Hidráulica }\end{array}$ & $\begin{array}{c}\text { Fm. Botucatu } \\
(\mathbf{m} / \text { dia })\end{array}$ & $\begin{array}{c}\text { Fm. Pirambóia } \\
(\mathbf{m} / \mathbf{d i a})\end{array}$ \\
\hline mínimo & 0,26 & 0,01 \\
máximo & 10,07 & 29,38 \\
média aritmética & 3,60 & 2,79 \\
média geométrica & 2,91 & 2,34 \\
desvio-padrão & 2,17 & 4,64 \\
\hline
\end{tabular}

Fonte: Elaborado pelos autores

É importante notar que a média geométrica dos valores de $\mathrm{K}$ obtidos nas formações Pirambóia e Botucatu, respectivamente 2,34 m/dia e 2,91 m/dia, são próximos àquela determinada pelo teste de bombeamento no poço $\mathrm{P} 2$, isto é, 1,97 $\mathrm{m} / \mathrm{dia}$. Este fato denota que a técnica para obtenção de K utilizada neste trabalho produz valores consistentes e realistas de condutividade hidráulica. Desse modo, os valores de $\mathrm{K}$ obtidos pelos testes de bombeamento podem ser interpretados como uma média geométrica dos valores de $\mathrm{K}$ de toda a seção filtrante do poço testado. É importante destacar que os valores de $\mathrm{K}$ obtidos no poço estão dentro do intervalo de valores típicos descritos na literatura (Tabela 3). 
Tabela 3 - Intervalos de valores de condutividade hidráulica do SAG determinados por testes de bombeamento, conforme diversos autores

\begin{tabular}{cc}
\hline $\begin{array}{c}\text { Intervalo de } \mathbf{K} \\
\text { (m/dia) }\end{array}$ & Trabalho \\
\hline $1,7-8,5$ & Araújo et al. (1999) \\
$0,4-4,0$ & Hirata et al. (2011) \\
$0,12-19,92$ & Gómez et al. (2010) \\
$0,46-0,70$ & Wahnfried et al. (2018) \\
$0,35-0,78$ & Hirata et al. (2012) \\
\hline
\end{tabular}

Fonte: Elaborado pelos autores

Além de produzir valores consistentes de $\mathrm{K}$, o método proposto possibilita a avaliação desses valores com elevado grau de resolução espacial em poços profundos. Tendo em vista que a perfilagem geofísica representa uma ferramenta comumente empregada em poços profundos, a metodologia ora proposta oferece um importante avanço para o entendimento das variações do $\mathrm{K}$ em subsuperfície.

\section{CONCLUSÕES}

Os resultados obtidos sugerem que a estimativa de permeabilidade fornecida pela equação de Kozeny-Carman, empregando dados de porosidade estimados a partir de perfilagens geofísicas, produz valores consistentes para as condições geológicas avaliadas, no caso, o Sistema Aquífero Guarani. Conclui-se, portanto, que os valores de porosidade estimados a partir da perfilagem sônica podem ser empregados para estimativas do parâmetro de condutividade hidráulica. A comparação com os testes de bombeamento indica que o valor de condutividade hidráulica obtido a partir da relação transmissividade/espessura representa a média geométrica dos valores de condutividade hidráulica de todo o perfil avaliado. Os testes preliminares aqui descritos demonstram a potencialidade do método utilizado e abrem a possibilidade da obtenção de valores de K, com elevado nível de resolução espacial, a partir de perfis geofísicos comumente empregados em poços profundos. 


\section{REFERÊNCIAS}

ARAÚJO, L. M.; FRANÇA, A. Barros; POTTER, P. E. Hydrogeology of the Mercosul aquifer system in the Paraná and Chaco-Paraná Basins, South America, and comparison with the Navajo-Nugget aquifer system, USA. Hydrogeology Journal, v. 7, n. 3, p. 317-336, 1999. https://doi.org/10.1007/s100400050205

CARMAN, P. C. Flow of gases through porous media, Butterworths Scientific Publications, London. 1937.

CARMAN, P. C. The determination of the specific surface of powders. Trans. Inst. Chem. Eng. (London), v. 15, p. 150, 1956.

CAETANO-CHANG, Maria Rita; WU, Fu Tai. Arenitos flúvio-eólicos da porção superior da Formação Pirambóia no centro-leste paulista. Revista Brasileira de Geociências, v. 36, n. 2, p. 296-304, 2006. https://doi.org/10.25249/0375-7536.2006362296304

CARRIER III, W. David. Goodbye, hazen; hello, kozeny-carman. Journal of Geotechnical and Geoenvironmental Engineering, v. 129, n. 11, p. 1054-1056, 2003.

https://doi.org/10.1061/(ASCE)1090-0241(2003)129:11(1054)

DEWANDEL, Benoît et al. Upscaling and regionalizing hydraulic conductivity and effective porosity at watershed scale in deeply weathered crystalline aquifers. Journal of Hydrology, v. 416, p. 83-97, 2012. https://doi.org/10.1016/j.jhydrol.2011.11.038

FETTER, Charles Willard. Applied hydrogeology. Waveland Press, 2018.

FREUND, D. Ultrasonic compressional and shear velocities in dry clastic rocks as a function of porosity, clay content, and confining pressure. Geophysical Journal International, v. 108, n. 1, p. 125-135, 1992. https://doi.org/10.1111/j.1365-246X.1992.tb00843.x

GIANNINI, Paulo César Fonseca et al. Paleoventos e paleocorrentes subaquosas do sistema deposicional Pirambóia nos estados de São Paulo e Paraná, Bacia do Paraná: estudo baseado em análise estatística de dados azimutais. Revista Brasileira de Geociências, v. 34, n. 2, p. 282-292, 2004. https://doi.org/10.25249/0375-7536.2004342282292

GÓMEZ, Andrea A.; RODRÍGUEZ, Leticia B.; VIVES, Luis S. The Guarani Aquifer System: estimation of recharge along the Uruguay-Brazil border. Hydrogeology Journal, v. 18, n. 7, p. 1667-1684, 2010. https://doi.org/10.1007/s10040-010-0630-0

HAN, De-hua; NUR, Amos; MORGAN, Dale. Effects of porosity and clay content on wave velocities in sandstones. Geophysics, v. 51, n. 11, p. 2093-2107, 1986. https://doi.org/10.1190/1.1442062

HIRATA, Ricardo et al. Relation between sedimentary framework and hydrogeology in the Guarani Aquifer System in São Paulo state, Brazil. Journal of South American Earth Sciences, v. 31, n. 4, p. 444-456, 2011. https://doi.org/10.1016/j.jsames.2011.03.006 
HIRATA, Ricardo et al. Explotação do Sistema Aquífero Guarani em Araraquara. Geologia USP. Série Científica, v. 12, n. 2, p. 115-127, 2012. https://doi.org/10.5327/Z1519874X2012000200008

HÖYNG, Dominik et al. High-resolution aquifer analog of fluvial-aeolian sediments of the Guarani aquifer system. Environmental earth sciences, v. 71, n. 7, p. 3081-3094, 2014. https://doi.org/10.1007/s12665-013-2684-5

KOPONEN, A.; KATAJA, M.; TIMONEN, J. Permeability and effective porosity of porous media. Physical Review E, v. 56, n. 3, p. 3319, 1997. https://doi.org/10.1103/PhysRevE.56.3319

KOZENY, J. Ueber kapillare leitung des wassers im boden, Wien, Akad. Wiss, v. 136, p. 271, 1927.

MCGREGOR, R. The effect of rate of flow on rate of dyeing II-The mechanism of fluid flow through textiles and its significance in dyeing. Journal of the Society of Dyers and Colourists, v. 81, n. 10, p. 429-438, 1965. https://doi.org/10.1111/j.1478-4408.1965.tb02615.x

MCCALL, W.; CHRISTY, T. M. Development of a hydraulic conductivity estimate for the Hydraulic Profiling Tool (HPT). In: The 2010 North American Environmental Field Conference \& Exposition: Conference Program with Abstracts: Session VII. 2010.

PANDA, Manmath N.; LAKE, Larry W. Estimation of single-phase permeability from parameters of particle-size distribution. AAPG bulletin, v. 78, n. 7, p. 1028-1039, 1994. https://doi.org/10.1306/A25FE423-171B-11D7-8645000102C1865D

PAPE, Hansgeorg; CLAUSER, Christoph; IFFLAND, Joachim. Variation of permeability with porosity in sandstone diagenesis interpreted with a fractal pore space model. In: Fractals and dynamic systems in geoscience. Birkhäuser, Basel, 2000. p. 603-619.

https://doi.org/10.1007/978-3-0348-8430-3 8

RAGHAVAN, Rajagopal. A review of applications to constrain pumping test responses to improve on geological description and uncertainty. Reviews of Geophysics, v. 42, n. 4, 2004. https://doi.org/10.1029/2003RG000142

ROVEY, Charles W.; CHERKAUER, Douglas S. Scale dependency of hydraulic conductivity measurements. Groundwater, v. 33, n. 5, p. 769-780, 1995. https://doi.org/10.1111/j.17456584.1995.tb00023.x

SCHULZE-MAKUCH, Dirk et al. Scale dependency of hydraulic conductivity in heterogeneous media. Groundwater, v. 37, n. 6, p. 904-919, 1999. https://doi.org/10.1111/j.17456584.1999.tb01190.x

SUN, Kerang. Formulating surrogate pumping test data sets to assess aquifer hydraulic conductivity. Journal of Hydrology X, v. 1, p. 100004, 2018.

https://doi.org/10.1016/j.hydroa.2018.100004

THEIS, Charles V. The relation between the lowering of the piezometric surface and the rate and duration of discharge of a well using ground-water storage. Eos, Transactions American Geophysical Union, v. 16, n. 2, p. 519-524, 1935.https://doi.org/10.1029/TR016i002p00519 
VERNIK, L. Predicting lithology and transport properties from acoustic velocities based on petrophysical classification of siliciclastics. Geophysics, v. 59, n. 3, p. 420-427, 1994. https://doi/10.1190/1.1443604

WAHNFRIED, Ingo et al. Anisotropia e confinamento hidráulico do Sistema Aquífero Guarani em Ribeirão Preto (SP, Brasil). Geologia USP. Série Científica, v. 18, n. 3, p. 75-88, 2018. https://doi.org/10.1190/1.1443604

WU, Fu-Tai; CAETANO-CHANG, Maria Rita. Estudo mineralógico dos arenitos das formações Pirambóia e Botucatu no Centro-Leste do Estado de São Paulo. Revista do Instituto Geológico, v. 13, n. 1, p. 58-68, 1992. https://doi.org/10.11606/issn.2316-9095.v18-128529

WYLLIE, Malcolm Robert Jesse; GREGORY, Alvin Ray; GARDNER, Louis Wright. Elastic wave velocities in heterogeneous and porous media. Geophysics, v. 21, n. 1, p. 41-70, 1956. https://doi.org/10.1190/1.1438217

ZHOU, Yijie; HOSSAIN, Zakir. Permeability-velocity model for clay bearing and cemented sandstone. In: SEG Technical Program Expanded Abstracts 2014. Society of Exploration Geophysicists, 2014. p. 2941-2946. https://doi.org/10.1190/segam2014-0774.1 